\title{
TÁCTICAS EN VEJIGA
}

\author{
José Vicente Rodríguez
}

Fundación Puigvert Barcelona. Barcelona. España.

\section{ÍN DICE / RESUMEN}

1. Límites en la RTU de los tumores vesicales superficiales.

J. Vicente Rodríguez.

2. Cirugía endoscópica del tumor vesical profundo: ¿cuándo? ¿cómo?

J. Vicente Rodríguez.

3. Biopsias randomizadas en tumores vesicales (BR): ¿cuando? ¿por qué?

J. Vicente Rodríguez.

4. La segunda biopsia (Re-RTU) en tumor vesical superficial: ¿siempre necesaria?

J. Vicente Rodríguez.

5. Solución endourológica del divertículo vesical: ¿posible?

J. Vicente Rodríguez.

Palabras clave: Tácticas en vejiga. Tumor vesical. Biopsia randomizada. Cirugía endoscopica. RTU.

José Vicente Rodríguez

Fundación Puigvert Barcelona.

C/ Cartagena 340-350

08025 Barcelona (España)

\section{1. ĹMITES EN LA RTU DE LOS TUMORES VESI- CALES SUPERFICIALES.}

J. Vicente Rodríguez.

Los posibles límites de la cirugía endoscópica dependen de cuanto es posible / conveniente resecar y hasta cuando repetirla.

\section{¿Hasta "cuanto" es posible / conveniente resecar?}

El dominio técnico, la táctica de resección y la paciencia del cirujano, establecen los límites de cuanto se puede resecar, tanto en los tumores de gran volumen ( $>3 \mathrm{~cm}$ o sumatorio de varios tumores $>20 \mathrm{gr}$ ), como la papilomatosis (tumores ocupando el 70-80\% de la mucosa vesical).

En los tumores únicos de gran volumen, la RTU debe ser secuencial y ordenada: de derecha a izquierda, de atrás adelante. Si se altera el orden las franjas ocultan la parte resecada y se prolonga o dificulta la resección de la masa tumoral.

En la papilomatosis la resección debe iniciarse por el tumor "más difícil, mas alto, más sangrante". Se comienza en la cara anterior (cúpula) por ser la más difícil y alta (se evita la acumulación de burbujas); a continuación se aborda el hemicontorno inferior identificando (cromoscopia) los meatos ureterales, dejándose las paredes posterolaterales para el final de la intervención.

En esta, se admite la cirugía endoscópica en varios tiempos. En nuestra experiencia: $55 \%$ en un solo tiempo (media de RTU de 56 gramos), el $44 \%$ en dos 
TABLA I. COMPUCACIO NES (En 61 casos de RTU / MacroTm)

\begin{tabular}{|l|l|l|l|l|l|}
\hline C. Peroperatorias & N o casos & $\%$ & C. Postoperatorias & N o casos & $\%$ \\
\hline Sangrado & 13 & 21.3 & Reflujo vesico/ ureteral & 11 & 18 \\
\hline Perforación & 3 & 4.9 & Estenosis uretral & 5 & 8.2 \\
\hline Reoperación urgente & 8 & 13 & Retracción vesical & 2 & 3.3 \\
\hline
\end{tabular}

tiempos (media $26 \mathrm{gr}$ )y el $2 \%$ en tres tiempos (media $14 \mathrm{gr}$ ).

Esta cirugía no está exenta de complicaciones, como lo demuestra nuestra experiencia en 61 casos de RTU de tumores vesicales de gran volumen / papilomatosis y que se reflejan en la Tabla I.

A sí pues la cirugía endoscópica de los tumores vesicales de gran volumen / papilomatosis, es posible con táctica y técnica depuradas, pero asumiendo la posible cirugía en dos tiempos y el no despreciable índice de complicaciones.

A demás de posible, la cirugía endoscópica en estas condiciones debe de ser "conveniente".

Su utilidad viene determinada por como evoluciona:

- Progresión: en nuestra experiencia no progresó ningún caso de TaG 1, el $26 \%$ de los T1G $1-2$ y el $54 \%$ de los G 3 T1.

- Mortalidad: muertos por cáncer 18\%, muertos sin cáncer $6.5 \%$, vivos con/ sin cancer $67,5 \%$.
- Necesidad de cistectomía: 16\%; se pudo conservar la vejiga en el $84 \%$ de los casos.

\section{¿Hasta cuando repetir la RTU?}

La RTU del tumor vesical superficial, aislada 0 asociada a quimio y/ o inmunoterapia, tiene finalidad curativa; por consiguiente debe repetirse hasta que las condiciones vesicales o la evolución tumoral obliguen a cambiar de actitud.

La cistectomía se cuestiona cuando el tumor vesical progresa, cuando se asocia a CIS que no responde (dos ciclos de BCG), cuando la clínica miccional condiciona la calidad de vida ( sufrimiento vesical, vejiga retráctil) o en caso de papilomatosis no tratable endoscópicamente.

En experiencia de Fundació Puigvert tanto en la serie clásica, como en la era de la BCG, las principales causas de cistectomía en la evolución de tumores vesicales superficiales vienen reflejado en la Tabla II.

TABLA II. CISTECTO MÍA EN TUMO RES VESICALES SUPERFICIALES

\begin{tabular}{|l|l|l|}
\hline Causa determinante (\%) & Serie clasica (167c) & Serie en era de BCG (43c) \\
\hline Sufrimiento vesical & $44 \%$ & $11.6 \%$ \\
\hline CIS a sociado recidivante & $43 \%$ & $64.7 \%$ \\
\hline Papilomatosis no controlable con cirugía endoscópica & $5 \%$ & $6.9 \%$ \\
\hline
\end{tabular}


En resumen, nuestros resultados sugieren que la RTU de tumores de gran volumen / papilomatosis es un procedimiento difícil, con alto índice de morbilidad y reoperación, pero su realización es útil y permite la conservación vesical en un elevado número de pacientes a los cuales se les debería realizar cistectomía para poder controlar su enfermedad. Los límites de la RTU por el tamaño o número tumorales, los pone el dominio técnico y la táctica quirúrgica apropiada.

La repetición de la RTU de los tumores vesicales superficiales, debe ser la norma, estableciendo su límite cuando deja de ser superficial, se asocia a CIS no respondedor o bien el sufrimiento / retracción vesical condiciones la calidad de vida de los pacientes.

\section{BIBUOGRAFÍA RECOMENDADA}

1. VICENTE, R.: "Tratamiento endoscópico en Tumores vesicales superficiales". Ed. J. Vicente, G. Chéchile, J. Salvador. Editorial Acción Médica, 121, 2000.

\section{CIRUGÍA ENDOSCÓPICA DEL TUMOR VESI- CAL PROFUNDO. ¿CUÁNDO? ¿CÓMO?}

J. Vicente Rodríguez.

La táctica terapéutica de los tumores vesicales infiltrantes (T.V.I.), debe incluir actualmente la cirugía endoscópica, como opción curativa con intento de preservación vesical.

El tratamiento endourológico: RTU radical, tiene demostrada su capacidad resolutiva dada la ausencia de tumor (TO) en el $10-14 \%$ de casos de cistectomía post RTU y en la supervivencia similar entre tumores vesicales $\mathrm{T} 2$ tratados por cistectomía y los tratados con RTU aislada (1)(2) o RTU mas quimioterapia (3)(4). La diferencia entre ambas opciones es que la cirugía endoscópica consigue entre un $45-65 \%$ de casos conservar la vejiga, con micción uretral y sexualidad normales.

Sin embargo el número de pacientes tributarios de esta cirugía es reducido, dada su estricta selección (¿CUANDO ?), su dificultad técnica (¿COMO?) y su seguimiento exigente y prolongado (4).

\section{¿CUÁNDO?}

Para incluir un paciente con tumor vesical infiltrante en régimen de preservación vesical mediante RTU radical / quimioterapia, debe darse estas características:

- Tumor infiltrante T2 (diferenciado por el patólogo en el estudio de resección seriada). A usencia de CIS múltiple asociado (según biopsias randomizadas).

- Tumor único, franjeado/ aframbuesado, menor de 3-

$4 \mathrm{~cm}$ de diámetro mayor (según visisón endoscópica).

- Ausencia de repercusión del aparato urinario superior (según exploración ecográfica previa).

- No evidencia de $\mathrm{N}+\mathrm{O}$ M o segundo tumor (según TAC previo).

- Condiciones de "operabilidad" (según riesgo quirúrgico, niveles de hemoglobina, etc.)

\section{¿CÓMO?}

La cirugía endoscópica del tumor infiltrante, con las características limitantes descritas, debe ser minuciosa y su dificultad estriba en el reconocimiento de las estructuras según los niveles de resección. En el tumor infiltrante el estadiaje biópsico o la curabilidad van a determinarse de acuerdo con la descripción macroscópica del reseccionista y el examen anatomopatológico del material resecado. En nuestra experiencia (5), en 118 casos de RTU radical en TVI como estadiaje, en el $56 \%$ el patólogo pudo determinar si el tumor resecado era T2 (52c) o T3 ( 14c).

La RTU radical con finalidad terapéutica se realiza de forma secuencial, ordenada y progresiva obteniendo fragmentos que se envían al patólogo por separado. Esta técnica está esquematizado en la Figura (6).

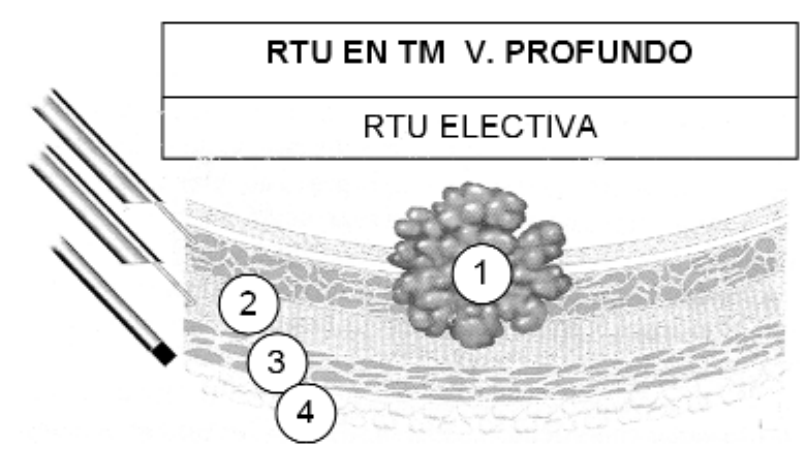


1. (masa tumoral) Resección de la masa exofítica tumoral hasta descubrir las fibras musculares superficiales.

2. (muscular superficial) El aspecto de superficie ofrece entramado de fibras sonrosadas / denso; al resecar este plano las fibras pierden firmeza y uniformidad.

3. (muscular profunda). Las fibras se espacian y entre ellas se insinúa tejido amarillento, la resección de este plano nos sitúa en el tejido graso perivesical.

4. (grasa) Se cambia el resector por el panendoscopio y se procede a obtener, con pinzas endoscópicas, dos o tres muestras biópsicas del tejido graso.

$\mathrm{N}$ osotros realizamos en los pacientes con idea de preservación vesical nueva cistoscopia + biopsias randomizadas + RTU de la zona cicatrizal, una vez finalizadas tres ciclos de carbomin o si no se asocia quimio a las tres semanas de la primera resección; hemos encontrado tumor residual en el $19 \%$ de casos.

Sin duda la RTU radical y la confirmación de ausencia de tumor residual en la re-RTU, es una forma de seleccionar los tumores infiltrantes de buen pronóstico.

La táctica electiva y técnica de estos casos consigue la conservación de la vejiga, con supervivencia similar a la conseguida con cirugía radical abierta (cistectomía).

\section{BIBUOGRAFÍA RECOMENDADA}

1. HERR, H.: "Transurethral resection of muscle-invasive bladder cancer: 10 year outcome". J. Clin. Oncol., 19: 89, 2001.

2. SOLSONA, E.; IBARRA, I.; RICOS, J.V. y cols.: "Feasibility of transurethral resection for muscle infiltrating carcinoma of bladder long-term follow-up of a prospective study". J. Urol., 159: 95, 1998.

3. GIVEN, R.W.; PEARSONS, J.T.; Mc CARLEY, D. y cols.: "Management and prognosis of transitional cell carcinoma superficial recurrence in muscle-invasive bladder cancer after bladder preservation". Eur. Urol., 44: 222, 2003.

4. VICENTE, J.; MONTELLEÓ, M.; LAGUNA, P. y cols.: "RTU de estadiaje del tumor vesical infiltrante". Act. Fund. Puigvert, 15: 186, 1996.

5. LAGUNA, P.; VICENTE, J.: "Cirugía endoscópica de la vejiga." Tratado de Endourología. J. Vicente Ed. Pulso. 4: 237, 1996.

\section{BIOPSIAS RANDOMIZADAS (BR): CUANDO Y POR QUÉ}

J. Vicente Rodríguez.

Es conocido el riesgo del urotelio de presentar lesiones premalignas/ CIS no detectables por técnicas de imagen ni por la exploración endoscópica, las BRs serían capaces de diagnosticar estas lesiones "no visibles"; así ha sido refrendado por extensa bibliografía.

Sin embargo, otros estudios las han cuestionado porque la displasia severa/ CIS pueden ser fácilmente identificado por citología y porque es escaso el $\mathrm{n}$ o de estas lesiones en las BRs (1). El grupo holandés (2), aparte dudar de su utilidad en la toma de decisiones terapéuticas, critican la mayoría de los trabajos publicados por escaso número de pacientes, corto seguimiento y factores pronósticos sin valoración con análisis multivariante.

$\mathrm{N}$ osotros (3) hemos realizado un estudio que cumple todas las exigencias científicas: 1.529 tumores superficiales iniciales sometidos a RTU + BR (cinco en vejiga más una en uretra si varones), seguimiento medio de 4,2 años y estudio realizado mediante análisis multivariante usando regresión de Cox. Con ello hemos demostrado su facilidad técnica, inocuidad, fiabilidad y sobre todo su utilidad en demostrar CIS asociado, así como factor pronóstico de recurrencia y mortalidad, lo que nos ha servido de ayuda en la toma de decisiones terapéuticas (4).

Estos datos nos permiten responder a cuando (indicaciones) y porque (ayuda en orientación terapeutica), como queda resumido en la Figura 1.

FIG URA 1.

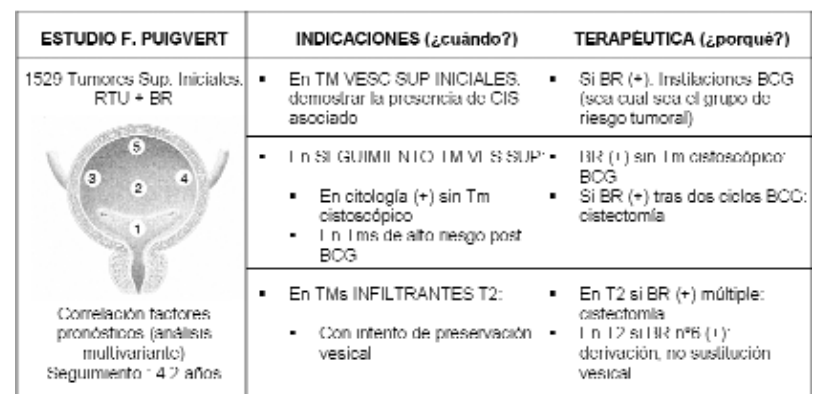




\section{¿CUANDO ESTÁN IN DICADAS LAS BR?}

En tumores vesicales superficiales iniciales: basadas en:

- Demostrar la presencia de displasia/CIS asociado. Este dato está ampliamente reflejado en la bibliografía: Flam: 24\%, Miltifi: 23\%, W itjes: $21.2 \%$. Cuando se valora solamente la presencia de CIS se reproducen porcentajes similares: Zein: $23 \%$, Solsona (5): 15,6\%, vicente (3): 19\%.

- Valorar la importancia de las BR (+) como factor pronóstico. En nuestra experiencia la $B R(+)$ (presencia de CIS) determinó un riesgo de recurrencia 1.6 veces mayor que en los casos en que fue negativa; similar para Flam: $\mathrm{BR}(+)$ : $63 \%$ recidivas y $\mathrm{BMN}(-)$ : $27 \%$; W itjes(2). no encuentra diferencias en recurrencias ni progresión en casos con BR(+) o BR (-). En nuestro estudio (4) las $B R(+)$ tuvieron 3.24 veces más riesgo de muerte que cuando eran negativas.

\section{En el seguimiento de tumores vesicales:}

- Las BR están indicadas en los casos de citología(+) en el seguimiento después de la RTU, sin que se demuestre por imagen, tumor en aparato urinario superior ni tumor vesical demostrable por visión endoscópica. Esta indicación está recomendada en las guidelines de la AEU.

- En el seguimiento de tumores vesicales de "alto riesgo"; tras instilaciones de inducción con BCG, en nuestra experiencia la confusión cistoscópica de los "tuberculomas" pseudotumorales y las altera ciones celulares en el estudio citológico, dificultan el diagnóstico de persistencia o nueva incidencia de CIS vesical (4).

\section{En la selección de Tms Infiltrantes T2, con intento de preservación vesical:}

Las BM s (+) (expresión de CSI múltiple) condiciona o contraindica el intento de preservación vesical y obliga a la cirugía radical.

\section{¿PO R Q UÉ? VALOR DE LAS BR EN EL PLANTEAM IEN - TO TERAPÉUTICO}

\section{En los tumores vesicales superficiales iniciales:}

Las BR (t) son determinantes en la decisión terapéutica: sea cual sea el riesgo tumoral, debe iniciarse las instilaciones con BCG; el inicio precoz de la inmunoterapia precoz disminuye el riesgo de evolución adversa.

\section{En el seguimiento de los tumores vesicales:}

- La BR (+) en casos de ausencia de tumor de AUS o tumor vesical endoscópico (alarma creada por citología positiva en el seguimiento después de RTU tumoral), orienta a la instauración de tratamiento con instilaciones endovesicales de BCG.

- La persistencia de CIS =BMN (+), después de dos ciclos de BCG, resulta en tumores de alto riesgo, un valor predictivo fiable para decidir la cistectomía total.

- La BR (+) en la biopsia n6 (uretra prostática) en casos con indicación de cistectomía, orienta en la elección de la técnica radical: derivación urinaria y no sustitución vesical.

\section{En la evaluación inicial de tumores infiltrantes T2:}

La prioridad de las BR (CIS +), condiciona la tentativa de preservación vesical: si $\mathrm{BR}(+)$ única reevaluar la decisión según respuesta de inmunoterapia intravesical; si BR (+) múltiple descartar la preservación definitivamente.

\section{BIBUOGRAFÍA RECOMENDADA}

1. VAN MIJDEN, A.; OOSTERLINK, W.; SYLVESTER, R.: "Members of the EORTC-GU Group superficial bladder committee: the significance of bladder biopsies in Ta, T1 bladder tumors". Report from the EORTC-GU Group. J. Urol., 157: 838, 1997.

2. KEMENEY, L.; WITJES, J.A.; HEIJBROCK, R. y cols.: "Should random urotelial biopsies be taken from patients with primary superficial bladder cancer?". Br. J. Urol., 73: 164, 1994.

3. VICENTE, R.: "Biopsias randomizadas: experiencia y criterio de la Fundación Puigvert. En tumores vesicales superficiales". Ed. J. Vicente, G. Chéchile, J. Salvador. Editorial Acción Médica,73, 2000.

4. VICENTE, J.: "Utilidad de las biopsias múltiples normatizadas (BMN) en el planteamiento terapéutico del carcinoma vesical". Tratado de Oncología Urológica. Ed. L. Resel. J. Moreno E. Saned S. L. Madrid, 41: 539, 2003.

5. SOLSONA, E.; IBORRA, I.; RICOS, J. y cols.: "Car cinoma in situ associated with superficial bladder tumor". Eur. Urol., 19: 93, 1991. 


\section{LA SEGUNDA BIOPSIA (Re-RTU) EN TM SUPERFICIAL ¿SIEMPRE NECESARIA?}

J. Vicente Rodríguez.

La RTU repetida entre la 1a y 6 a semanas después de la cirugía endoscópica inicial, tiene como finalidad detectar tumor residual; la exploración cistoscópica antes 0 a los tres meses de la RTU inicial pretende demostrar la recurrencia o presistencia tumoral precoz. A mbas circunstancias están relacionadas con la ejecución técnica de la RTU tumoral inicial.

En las G uidelines de la A EU se recomienda la obtención de fragmentos biópsicos separados según profundidad de la resección tumoral. Bressel propuso en el pasado siglo la "RTU fragmentada" del Tm vesical, con la que Klain (1991) consiguió reducir en $20.3 \%$ la incidencia de tumor residual. Nosostros (1) hemos realizado rutinariamente la RTU secuencial:

1. Coagulación de vasos nutricios.

2. RTU de masa tumoral.

3. RTU de la masa muscular subyacente.

4. Electrocoagulación de la base de resección y un centímetro alrededor.

Es indiscutible que la realización correcta y completa de la RTU tumoral inicial asegura el etiquetaje biópsico tumoral (Tm superficial o profundo) e influye en la existencia de tumor residual (segunda RTU) o recurrencia precoz (3 meses).
En 153 pacientes sometidos a RTU "completa tumoral", Mulders (1994), no pudo estadiar el tumor en el $22 \%$ de casos (2). W ijstrom (1998) en su estudio en el $41 \%$ de casos no pudo afirmar el estadio por falta de representación de muscular parietal (pTx). La incidencia de pTx fluctúa según autores: Herr el $39.6 \%$, en nuestra experiencia: $6.6 \%$ y para RUTT menos del $5 \%$.

- La segunda resección sistemática con fines biópsicos, realizada entre 1 y 6 semanas post RTU inicial, proporciona globalmente un alto porcentaje de tumor residual: Herr (1999): $72 \%$, 0 jea (2000): $56 \%$, Klan (1991): $43.5 \%$ y Schips (2002): 36\%. Sin embargo estudios recopilatorios 0 multicéntricos actuales, como los de 0 tto (3) y Brausi (4), encuentran una amplia pinza porcentual: entre 3 y $46 \%$; esto se justifica dado el número de variables que influyen en la incidencia del tumor residual en la segunda RTU: diferencias según el centro donde se realizó la primera intervención, dominio técnico y/ o tipo de tumor inicial. Ello queda reflejado en la Tabla I.

0 tro dato relacionable con la RTU inicial incompleta es la demostración cistoscópica de ¿persistencia? 0 recurrencia precoz del tumor vesical (3 meses).

Como en la segunda RTU, el porcentaje de casos de recurrencia a 3 meses varía según distintas eventualidades:

TABLA I.

\begin{tabular}{|c|c|c|}
\hline AUTOR & VARIABLE & \% DE TM RESIDUAL (TmR) EN LA 2 $\stackrel{\text { a }}{\text { RTU }}$ \\
\hline BRAUSI & SEG ÚN CENTRO & Varía entre el 3.4 y el $20.6 \%$ \\
\hline VOGLEI & & El 75\% de TmR en igual localización que el inicial \\
\hline $\begin{array}{l}\text { KA LO ZSY/ RG UEZ- } \\
\text { RUBIO }\end{array}$ & $\begin{array}{l}\text { SEGÚN TÉCNICA } \\
\text { EN LA 1ạ RTU }\end{array}$ & El $26-28.5 \%$ de TmR en base de la 1 a RTU tumoral \\
\hline HERR & & El $72 \%$ de TmR pero $40 \%$ pTx en la $1 \stackrel{\text { a RTU }}{ }$ \\
\hline MILLER/ KLAN & & El $12-35 \%$ de TmR en el borde de la 1a RTU \\
\hline KA LO ZSY-RG UEZ- & & Tm inicial: $\mathrm{Ta}$ Tm $\mathrm{R}$ en 20 RTU: $12.7 \%$ \\
\hline RUBIO & SEGUN TUMORES & Tm In. T1-Ta Tm R en 20 RTU:37.8\% \\
\hline KLAN & EN LA 1 a RTU & Tm Inicial: T1 Tm R en $2 \stackrel{0}{ } \mathrm{RTU}: 40 \%$ \\
\hline OJEA & & Tm In T1 múltiple Tm R en 20 RTU: $57 \%$ \\
\hline
\end{tabular}


Para Balincourt, en el estudio 30.911 de EO RTC, la recurrencia precoz fluctúa entre el 11 y el $60 \%$, según el centro hospitalario que aporta los datos al estudio (5). Brausi (6) llama la atención sobre la importancia cronológica en la variabilidad de resultados: en el periodo de 1975-1978 se detectan un promedio de $21-43.8 \%$ de recurrencia a tres meses y entre 1987-1989 de 3-5.3\%. También es una variable significativa el número de tumores a resecar en la cirugía inicial: en tumores únicos 3-2\% (Van Meijden), 8.7\% (Brausi), 4.2\% (Fundació Puigvert) y en tumores múltiples: 7-75\% (Van M eijden, 21-32\% (Brausi, 10.3 (F.P.) Nosotros (1) destacamos la diferencia del número (\%) de recurrencias precoces según grupos de riesgo. Grupo de bajo riesgo: $2 \%$, grupo intermedio: $4 \%$ y grupo de alto riesgo: $9.4 \%$

Dando respuesta a la pregunta inicial, con la suma de datos bibliográficos y nuestra propia experiencia, concluimos:

- La RTU inicial del tumor vesical superficial debe de realizarse secuencial, correcta y completamente; ello comporta la finalidad curativa, garantiza el estadiaje tumoral (pTX que no supere el $5 \%$ ) y reduce el porcentaje de tumor residual, la persistencia tumoral y la recurrencia precoz; en consecuencia limita la necesidad de la RTU repetida y la cistoscopia precoz.

- La segunda RTU biópsica no es necesaria en tumores vesicales superficiales de bajo e intermedio riesgo ( que representan casi el $80 \%$ de nuestra experiencia), sean únicos o múltiples, siempre que el cirujano responsable tenga certeza de haber realizado correcta y completamente la RTU tumoral inicial.

- La segunda RTU biópsica será necesaria en tumores de alto grado CIS (Pospuesta al final de la inmunoterapia asociada), en caso de citología positiva en el seguimiento inmediato post RTU; cuando el paciente ha sido resecado en otro centro y no tenemos información suficiente para decisión terapéutica y en casos en que el cirujano responsable no esté seguro 0 satisfecho de la RTU inicial.

- La valoración precoz de recurrencia tumoral, cistoscopia a tres meses, es innecesaria en tumores de bajo e intermedio riesgo dado el porcentaje escaso de recurrencias precoz: 2-4\%,con progresión casi inexistente: $0-0.4 \%$. Si debe realizarse en caso de tumores múltiples: recurrencia $10.3 \%$ y/ o tumores de alto riesgo: progresion de $9.4 \%$ sobre todo si la primera RTU fue dudosamente satisfactoria (1).

\section{BIBUOGRAFÍA RECOMENDADA}

1. VICENTE, J.: "Tratamiento endoscópico". Tumores vesicales superficiales. Ed J. Vicente, G. Chechile, J. Salvador. Editorial Acción Medica, 121, 2000.

2. JAKSE, G.; ALGABA, F.; MALMSTRÖN. y cols.: "A second look TUR in transitional cell carcinoma: why?”. Eur. Urol., 45: 539, 2004.

3. OTTO, T.; SUHR, J.; RÜBEN, H.: "Is there a need or an evidence for rebiopsing patients after a first TUR?". EAU Update Series, 1: 87, 2003.

4. BRAUZI, M.; COLLETE, L.; KURTH, K. y cols.: "EORTC: Varaibility in the recurrence rate at first follow up after TUR in stage $\mathrm{Ta} / \mathrm{T} 1$ transitionla cell carcinoma of the bladder: a combined analysis of seven EORTC studies". Eur. Urol., 41: 523, 2002.

5. RODRIGUEZ-RUBIO, F.J.; GARRIDO, S.; RIVAS, D. y cols.: "Segunda resección en pacientes con tumores vesicales Ta-T1”. Actas Urol. Esp., 25: 553, 2001.

6. BRAUSI, M.; KURTZ, K.H.; VNA DER MEIJDEN, A.P. y cols.: "Variability in the three months recurrence rate after TUR in superficial transitional cencer of bladder: a combined analysis of 8 EORTC studies". J. Urol., 159: 541, 1998.

\section{SOLUCIÓN ENDOUROLÓGICA DEL DIVERTÍCULO VESICAL ¿POSIBLE?.}

J. Vicente Rodríguez.

El divertículo vesical tributario de cirugía endoscópica, es el secundario a obstrucción infravesical, esté o no habitado por tumor en su interior.

En ambas circunstancias el diverticulo tiene como localización preferente la suprameática/ retrotrigonal, lo que facilita el abordaje endoscópico y su drenaje postquirúrgico.

La boca diverticular, frecuentemente estrecha, dificulta tanto la exploración como la actuación endoscópica. Las paredes diverticulares son finas, con escasa/ desordenada representación de fibras musculares, lo que facilita la perforación parietal y la penetración celular tumoral en profundidad. En a mbos casos la técnica endoscópica y el instrumental empleado son convencionales, resector con asas de incisión, corte y coagulación. Sin embargo, últimamente se ha sumado la diverticulectomía laparoscó- 
pica(1), que compite con la cirugía endourológica y la abierta (diverticulectomía) que es la más frecuentemente utilizada.

La táctica terapéutica endourológica está condicionada a las peculiaridades técnicas y a la cuidadosa elección de los casos tributarios de ella.

- La simultaneidad terapéutica desobstructiva por RTU y tratamiento endoscópico del divertículo secundario, constituyen una buena solución endoscópica en los casos idóneos, que permite evitar la cirugía en dos tiempos y las complicaciones infecciosas del postoperatorio. La técnica viene esquematizada en la Figura 5.

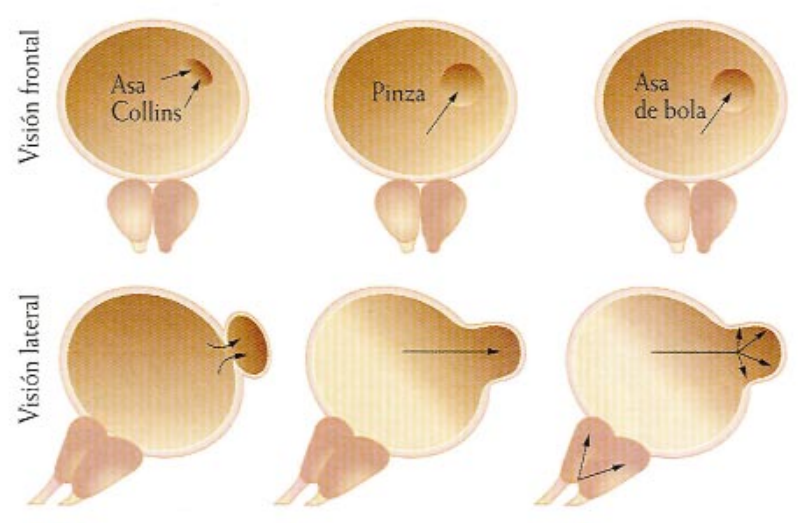

FIG URA 5.

La elección de casos para ser resueltos con esta técnica debe ser estricta: divertículos menores de tres centímetros de diámetro mayor, boca diverticular estrecha (menos de dos centímetros de separación ecográfica), y situados en paredes postero-laterales. En nuestra experiencia (2), reafirmada en estudios recientes (3), en divertículos menores de $5 \mathrm{~cm}$ de diámetro, el $78 \%$ desaparecieron ecográficamente, el $11 \%$ disminuyeron su tamaño mas del $50 \%$ y $11 \%$ persistieron sin variación.

El tumor intradiverticular, se asocia frecuentemente a tumor vesical; su incidencia es escasa: 1.3\% en nuestra experiencia, generalmente de mayor grado y estadio que los tumores vesicales superficiales en general (dada la delgadez de la pared del divertículo) y técnica endourológica condicionada y difícil.

La técnica endoscópica se inicia como en el esquema anterior: apertura de la boca diverticular y biopsia de la mucosa intradiverticular sumando la tumoral, antes de resecar o fotocoagular el tumor intradiverticular y finalizando con la RTU de tumores vesicales asociados (4).

Los casos de tumor intradiverticular en que está indicada la técnica endoscópica, se reducen a los casos de tumores de bajo grado y sin invasión de la lámina propia (Ta). Las series son escasas en número y seguimiento; las más representativas: M emorial SloanKatering de $\mathrm{N}$ ew York 39 pacientes (6) y Fundació Puigvert 34 pacientes (5). En ambas a largo seguimiento, el índice de recidivas es del 33\% pero en nuestra experiencia $(4,5)$ la tasa de progresión fue del $23.5 \%$ lo que obliga a contemplar con reservas la elección de la técnica y a seleccionar cuidadosamente los casos.

\section{BIBUOGRAFÍA RECOMENDADA}

1. PORPIGLIA, F.; TARABUZZI, R.; COSSU, M. y cols.: "Is laparoscopic diverticulectomy after transurethral resection of prostate safe and effective? Comparison with open surgery". J. Endourol., 18: 73, 2004.

2. VICENTE, R.: "Tratamiento endoscópico: RTU/incisión prostática en HBP 2001”. Ed. Vicente. Ed. Acción Médica. 4: 153, 2001.

3. SANTOMASTOSO, C.; OLIVA, A.: "Transurethral treatment of bladder diverticuli". Arch. Ital. Urol. Androl., 71: 275, 1999.

4. VICENTE, J.: "Cirugía Endoscópica de la próstata". Tratado de endourología. Ed. J. Vicente. Ed. Pulso, 5: 303, 1996.

5. CHECHILE, G.E.: "Tumores en divertículo vesical". Tumores vesicales superficiales. Ed. J. Vicente, G. Chechile, J. Salvador. Ed. Acción Médica, 269, 2000.

6. GELIJAMIN, A.; YOSSEPOWITH, O.; BECK, S.D. y cols.: "Carcinoma in a bladder divertículum: preservation and treatment outcome". J. Urol., 170: 1761, 2003. 